Results 93 children (74 females and 19 males) with cSLE were examined with median (range) follow up time of 7 (0.524) years and the median (range) age at diagnosis of 13 (519) years. Mean (SD) SLEDAI-2K was 18.3 (9.0) at the disease onset. $38 \%$ had organ damage at the last follow up with the median (range) SDI 0 (0-7). The first organ systems damaged in affected patients were renal (28\%), musculoskeletal (22\%), ocular (19\%), neuro-psychiatric (17\%), cardiovascular (11\%) and peripheral vascular $(2.8 \%)$, but with no significant statistical difference regarding the type of organ damage. Kendall rank correlation coefficient determined a positive correlation between SLEDAI-2K at the disease onset and SDI $(\tau b=0.252, p=0.003)$. There was no significant difference of SDI in regard to gender (Asymptotic Wilcoxon-Mann-Whitney Test, $\mathrm{p}=0.574)$. Using Kaplan-Meier method we estimated with $95 \%$ confidence the damage is not happening in the first 9 or 10 years after diagnosis or the occurrence of the first symptoms.

Conclusions The high correlation between SLEDAI-2K and SDI indicated that the presentation of the cSLE at onset can be prognostic of the course and long-term prognosis of lupus. Our findings suggest it is unlikely that organ damage will occur in $50 \%$ of patients in the first nine years of the disease course.

\section{P183 ARE QUALITY OF LIFE CONCERNS DIFFERENT BY SEX AMONG PATIENTS WITH SYSTEMIC LUPUS ERYTHEMATOSUS?}

${ }^{1}$ Mamatha Katikaneni, ${ }^{2}$ Meenakshi Jolly. 'Dept. of Rheumatology, Louisiana State University Health Sciences Center, Shreveport; ${ }^{2}$ Dept. of Rheumatology, Rush University, Chicago, USA

10.1136/lupus-2020-eurolupus.225
Background Not much is known about health outcomes among women, in comparison to men, in Systemic Lupus Erythematosus (SLE). Quality of life (QOL) is one of the core health outcome measures in SLE. This study compared health outcomes among SLE patients, stratified by sex, to facilitate better management strategies.

Methods Existent cross-sectional data from SLE patients from a multicenter health outcomes study was utilized. Demographic data, disease activity (SELENA-SLEDAI), damage (SLICC-SDI/ ACR), QOL (SF-36, LupusQoL) were compared by sex. Multivariate analysis (adjusted for age, disease activity and damage) were undertaken for QOL, using sex as the independent variable.

Results Of the 325 participants, 33 were men. Mean age was $41.9 \pm 13.0$ years, and over $53 \%$ were Caucasians. Mean SELENA-SLEDAI scores were $4.8 \pm 3.8$. There were no age, race or disease activity differences among the two groups. Damage was significantly greater among men than women. On univariate analysis, no differences in SF-36 QOL were observed by sex. However, women fared significantly worse than men in (Physical health, fatigue, Intimate relationship) LupusQoL domains. On multivariate analysis, female sex was an independent predictor of worse functioning on SF-36 (Physical function, vitality) and LupusQoL (Physical Health, Fatigue, Intimate Relationship) (table 1).

Conclusion SLE has differential effects on QOL among men and women. Use of disease specific QOL tool offers more comprehensive and disease pertinent evaluation of SLE impact. Women with SLE report worse impact on physical health, fatigue and intimate relationships. Further studies are required to better understand plausible reasons for these observations.

Abstract P183 Table 1
\begin{tabular}{|l|l|c|r|}
\hline & \multicolumn{2}{|c|}{ Multivariate* } \\
\hline Tool & Domain & \multicolumn{1}{|c|}{$95 \% \mathrm{Cl}$} & P value \\
\hline SF 36 & Physical Functioning & $-0.174-10.4,-1.19$ & 0.014 \\
& Physical Role Functioning & $-0.09-7.47,1.78$ & 0.227 \\
& Bodily Pain & $-0.07-6.79,2.16$ & 0.309 \\
& General Health Perceptions & $-0.09-7.70,1.77$ & 0.219 \\
& Vitality & $-0.18-10.30,-1.14$ & 0.015 \\
& Social Role Functioning & $-0.09-7.57,1.69$ & 0.212 \\
& Emotional Role Functioning & $-0.43-6.85,3.75$ & 0.564 \\
& Mental Health & $0.04-3.57,6.28$ & 0.588 \\
\hline LupusQoL & Physical Health & $-0.18-28.43,-2.72$ & 0.016 \\
& Emotional Health & $-0.02-12.64,9.09$ & 0.748 \\
& Body Image & $-0.13-25.01,1.85$ & 0.09 \\
& Pain & $-0.09-21.17,5.14$ & 0.231 \\
& Planning & $-0.11-23.36,3.32$ & 0.14 \\
& Fatigue & $-0.18-25.76,-2.82$ & 0.015 \\
& Intimate relationships & $-0.17-34.69,-2.9$ & 0.021 \\
& Burden & $-0.09-24.02,5.25$ & 0.207 \\
\hline
\end{tabular}

* Adjusted for age, activity and damage 